\section{Overcoming Severe XEDS Peak Overlaps with the AEM}

\author{
Scott D. Walck \\ South Bay Technology, Inc. \\ San Clemente, CA 92673 \\ walck@southbaytech.com
}

Of all the analytical techniques in electron microscopy, X-ray energy dispersive spectroscopy (XEDS) is perhaps the most useful. It provides a quick identification of the elements and even with semiquantitative methods; a reasonable composition can be obtained. However, in the scanning electron microscopy (SEM), there are materials systems in which severe peak overlaps of heavier elements $L$ and $M$ lines cannot be easily deconvolved with lighter elements' $K$ lines. In addition, without a sufficient overvoltage in the SEM, even identification of the heavier elements can be difficult. In the analytical electron microscope (AEM), there is always sufficient overvoltage to excite all of the elements' $K$-lines. However, all of the $K$-lines might not be able to be detected with commercially available instruments. This is illustrated in Fig.1 where the maximum energy of the detector system might be set to 10,20 , or $40 \mathrm{keV}$. Careful examination of Fig. 1 would indicate that in the AEM, the problems associated with elemental identification of the elements can be overcome because of the presence of the higher energy X-rays, particularly if the range of $40 \mathrm{keV}$ is used. However, there still exists the problem of a lighter element's $K$ peak overlapping with a lower energy $L$ or $M \mathrm{X}$-ray peak from a heavier element and thus preventing the integrated peak intensity for that line to be extracted. For quantification, the heavier element would use the higher energy $\mathrm{X}$-ray peak, but the lighter element's $K$ intensity is needed for the complete quantification.
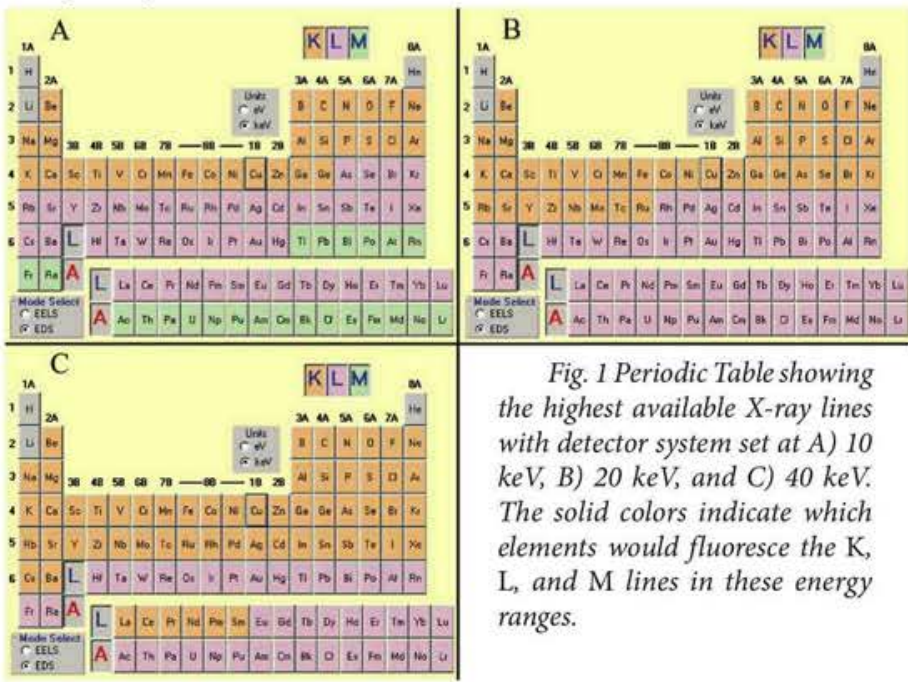

While working at the Materials Directorate at Wright Patterson Air Force Base, I was involved with the characterization of advanced tribological thin films used as solid lubricants. One material that looked promising as an adaptive lubricant was a pulsed laser deposited $\mathrm{PbO}-\mathrm{MoS}_{2} \cdot{ }^{1}$ An adaptive lubricant is one that adapts it properties to the environmental parameters such as temperature and atmosphere to provide continuous lubrication. The PLD PbO- $\mathrm{MoS}_{2}$ system held the possibility of offering the low temperature lubricant phase, $\mathrm{MoS}_{2}$, and the high temperature lubricant phases $\mathrm{PbO}$ or $\mathrm{PbMoO}_{4}$. In addition, different phases and microstructures would develop in the thin film depending on the processing that it was given. Identifying these phases proved to be difficult because of the peak overlaps of the S-Ka, Mo- $L a$, and $\mathrm{Pb}-\mathrm{Ma}$ lines as shown in Fig. 2. We were interested in the partitioning of these elements in the different phases that formed. The process that I used with the AEM will be discussed below.

What I would like to do in this article is to show how it is possible using an AEM to overcome the peak overlaps of a light element when the heavier elements' higher energy $\mathrm{X}$-rays are present. The process relies on two assumptions that are fairly reasonable. The first is that the partitioning of the $\mathrm{X}$-ray lines from a particular element is independent of the matrix, i.e. the composition and

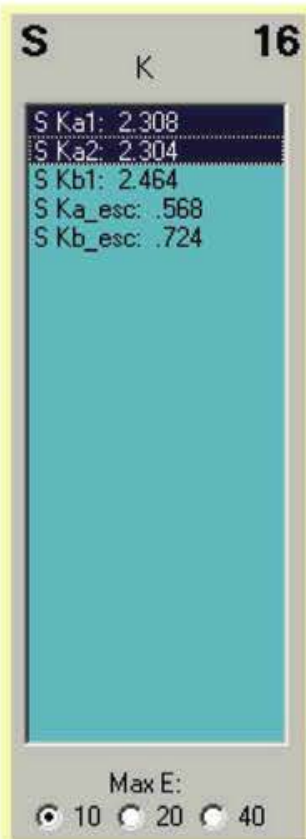

16 crystalline structure of the sample. For heavier elements that generate $L$ and $M$ lines, this is a very good assumption. To the analyst, what this means is that the ratio of the integrated peak intensities of a higher energy line to a lower energy line is constant. The second assumption is that the sample and all standards used in the analysis satisfy the thin film criteria for X-ray microanalysis. Following Williams ${ }^{2}$ and Zaluzec ${ }^{3}$, the thin film criteria is given as $\chi \rho t \leq 0.1$, where $\rho$ is the density of the sample, $t$ is the thickness of the sample, and $\chi=\mu / \rho \csc \psi$ and where
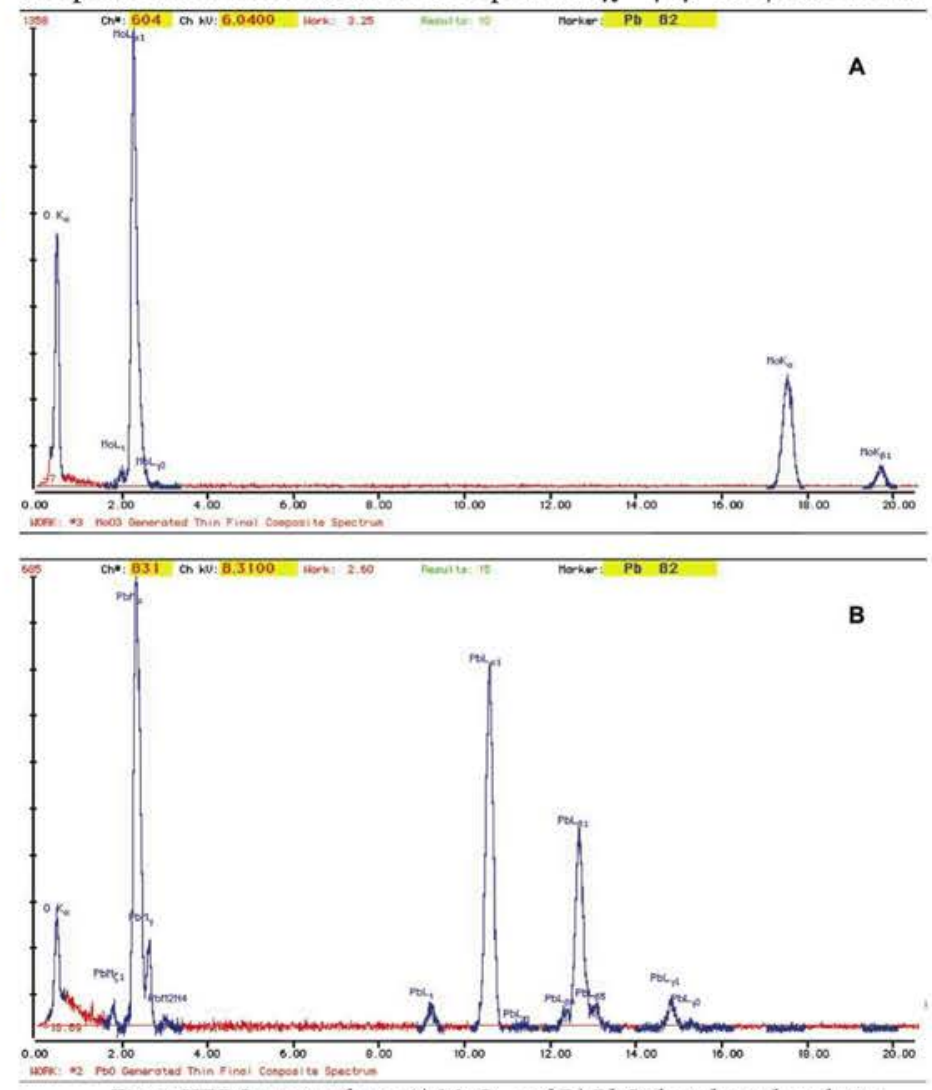

Fig. 3 XEDS spectra from A) $\mathrm{MoO}_{3}$ and $\mathrm{B}$ ) $\mathrm{PbO}$ that show the relative peak heights in a JEOL 2000FX TEM. 


\section{Multiphase Materials Characterization Fully Integrated...Totally Seamless}

Powerful Analytical Tools Optimized for Your SEM to Provide Results with Confidence

- Pegasus...Simultaneous data acquisitions for EDS (chemistry) and EBSD (crystallography)Includes the patented Chl-Scan software to improve multiphase analysis capability

- Neptune...Complimentary union of EDS and WDS for superior X-ray microanalysis-Improves quantification especially for low level concentrations

- Trident...Ultimate materials characterization system integrates EDS, EBSD, and WDS with no analytical performance compromise-Provides complete solutions for all your materials characterization requirements

\section{Results with Confidence}

As the world's leader in Electron Beam Microanalysis, EDAX continues to raise the performance standard with innovative features that are easy to adapt and provides results that you can trust to be complete and accurate.

For more information on our Seamless Integrated Systems for $E D S, E B S D$, and WDS visit our web site at www.EDAX.com/integration or call 1.201.529.4880 See us at PITTCON booth 3555!

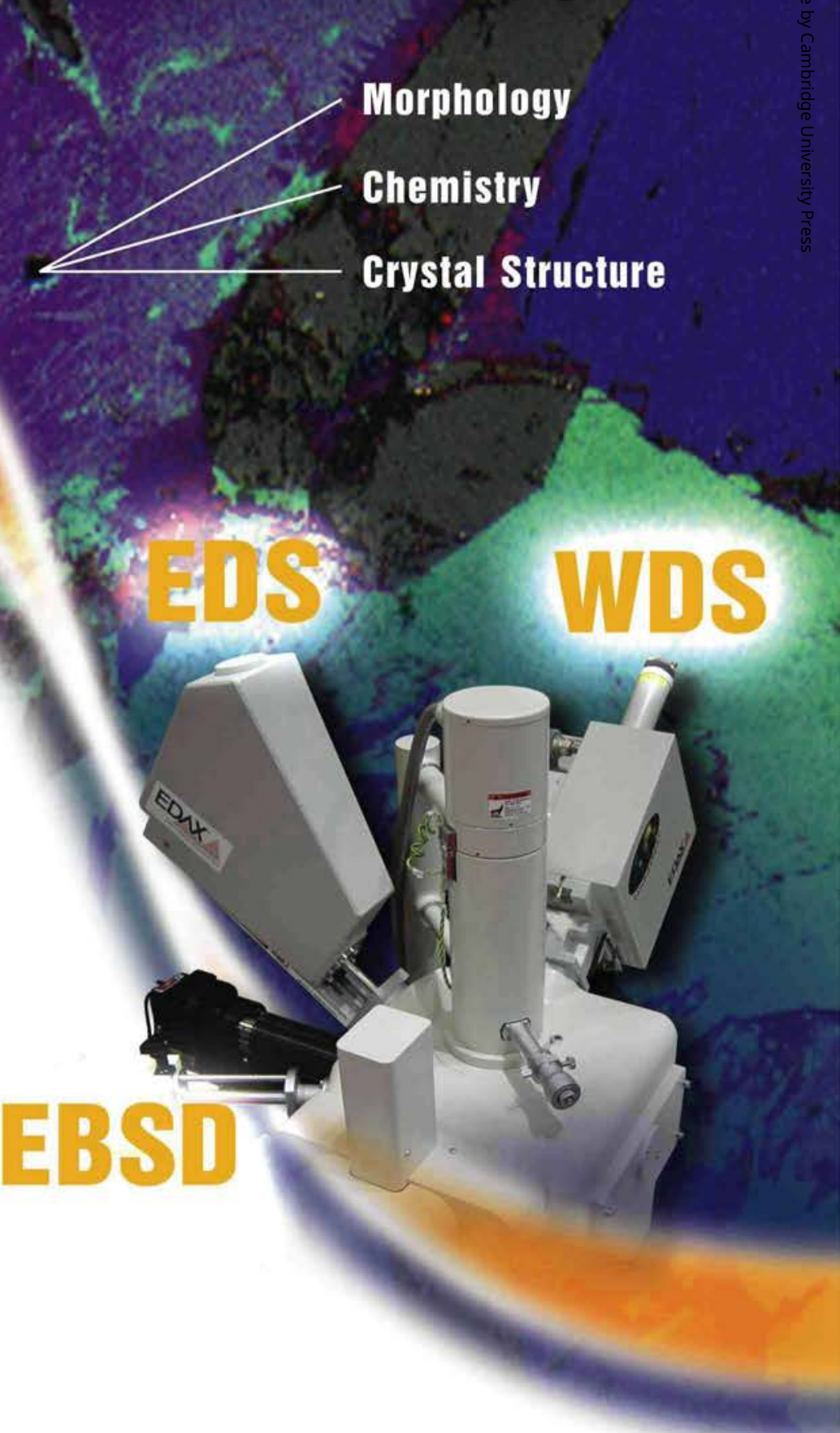


Table 1

Intensities, Ratio, K-factors, and Analysis Results

\begin{tabular}{|c|c|c|c|c|c|c|c|c|}
\hline \multicolumn{9}{|c|}{ Determination of Ratios } \\
\hline \multicolumn{4}{|c|}{ Mo } & & \multicolumn{4}{|c|}{$\mathrm{Pb}$} \\
\hline $\mathrm{I}(\mathrm{MOL})$ & I(MoKa) & $R(M o-L / K)$ & $1(\mathrm{OK})$ & & $\mathrm{I}(\mathrm{PbM})$ & $\mathrm{l}(\mathrm{Pb} \mathrm{La})$ & $R(P b-M / L)$ & $\mathrm{I}(\mathrm{OK})$ \\
\hline 19237 & 7048 & 2.73 & 7968 & & 23636 & 17905 & 1.32 & 4959 \\
\hline \multicolumn{9}{|c|}{ Determination of $\mathrm{K}$ factors $(\mathrm{Mo}, \mathrm{S})$ and $(\mathrm{Mo}, \mathrm{O})$} \\
\hline I(Mo Ka) & (MoL+SK) & $\mathrm{I}(\mathrm{M} \circ \mathrm{L})_{\text {calc }}$ & I(S K) & $\mathrm{k}(\mathrm{MOS})$ & $\mathrm{k}(\mathrm{MOO})_{\text {w/Abs }}$ & $\mathrm{k}(\mathrm{MOO})$ & $\mathrm{k}(\mathrm{OS})_{\mathrm{W} / \mathrm{Abs}}$ & $\mathrm{k}(\mathrm{OS})_{\text {cor }}$ \\
\hline 10425 & 63810 & 28454 & 35356 & 5.07 & 2.26 & 2.81 & 2.24 & 1.81 \\
\hline \multicolumn{9}{|c|}{ Determination of $\mathrm{K}$ factors $(\mathrm{Pb}, \mathrm{S})$ and $(\mathrm{Pb}, \mathrm{O})$} \\
\hline $\mathrm{I}(\mathrm{Pb} \mathrm{La})$ & $\mathrm{I}(\mathrm{Pb} \mathrm{M}+\mathrm{SK})$ & $\mathrm{I}(\mathrm{Pb} \mathrm{M}) \mathrm{calc}$ & I(S K) & $\mathrm{k}(\mathrm{PbS})$ & $\mathrm{k}(\mathrm{PbO})_{\text {W/Abs }}$ & $\mathrm{k}(\mathrm{PbO})_{\text {cor }}$ & $\mathrm{k}(\mathrm{OS})_{\text {W/Abs }}$ & $\mathrm{k}(\mathrm{OS})_{\text {cor }}$ \\
\hline 16991 & 35717 & 22429 & 13288 & 5.05 & 3.57 & 4.79 & 1.42 & 1.06 \\
\hline \multicolumn{9}{|c|}{ Stoichiometric PbO-MoS2 Intensity Data } \\
\hline & I(MoKa) & Calc I(MoLa) & I(PbLa) & Calc l(PbMa & I(Oka) & \multirow{2}{*}{\multicolumn{2}{|c|}{$\frac{\text { I(PbMa,MoLa,Ska) }}{86920}$}} & S by Sub \\
\hline & 9827 & 26822 & 20054 & 26473 & 6400 & & & 33625 \\
\hline
\end{tabular}

\begin{tabular}{|c|c|c|c|c|c|c|c|c|}
\hline \multicolumn{9}{|c|}{ Stolchlometric PbO-MoS2 Concentration Ratios C-L Analyzed from Generated Spectrum } \\
\hline $\mathrm{C}(\mathrm{Pb}) / \mathrm{C}(\mathrm{Mo})$ & $\mathrm{C}(\mathrm{Pb}) /$ & $\mathrm{C}(\mathrm{Pb}$ & $\begin{array}{c}\text { ACF } \\
\text { corrected }\end{array}$ & $\mathrm{C}(\mathrm{Mo}) / \mathrm{C}(\mathrm{S})$ & $\mathrm{C}(\mathrm{Mo}) / \mathrm{C}(\mathrm{O})$ & $\begin{array}{c}\text { ACF } \\
\text { corrected }\end{array}$ & 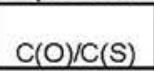 & $\begin{array}{c}\text { ACF } \\
\text { corrected }\end{array}$ \\
\hline 2.036 & 3.014 & 15.006 & 11.740 & 1.481 & 4.309 & 3.371 & 0.201 & 0.253 \\
\hline \multicolumn{9}{|c|}{ Stoichiometric PbO-MoS2 Concentration Ratios from Formula } \\
\hline 2.160 & 3.240 & 12.881 & & 1.497 & 5.952 & & 0.336 & \\
\hline \multicolumn{9}{|c|}{ Errors (Relative to Formula Generated Values) } \\
\hline $5.7 \%$ & $7.0 \%$ & $16.5 \%$ & $8.9 \%$ & $1.1 \%$ & $27.6 \%$ & $43.4 \%$ & $40.2 \%$ & $24.6 \%$ \\
\hline
\end{tabular}

$\mu / \rho$ is the mass absorption coefficient, and $\psi$ is the takeoff angle. In the case of a wedge sample, $\csc \psi$ would be replaced with the appropriate geometric terms, as discussed by Zaluzec. This assumption is usually the most critical for the X-rays overlapped with the light element $K$-line. For the analyst, it means that the sample and standards do not significantly absorb the X-rays. This is important because any absorption of the X-rays will change the ratio of the higher energy line to the lower energy line. This assumption is entirely dependent on the method of sample preparation of the sample and standards. However, with the

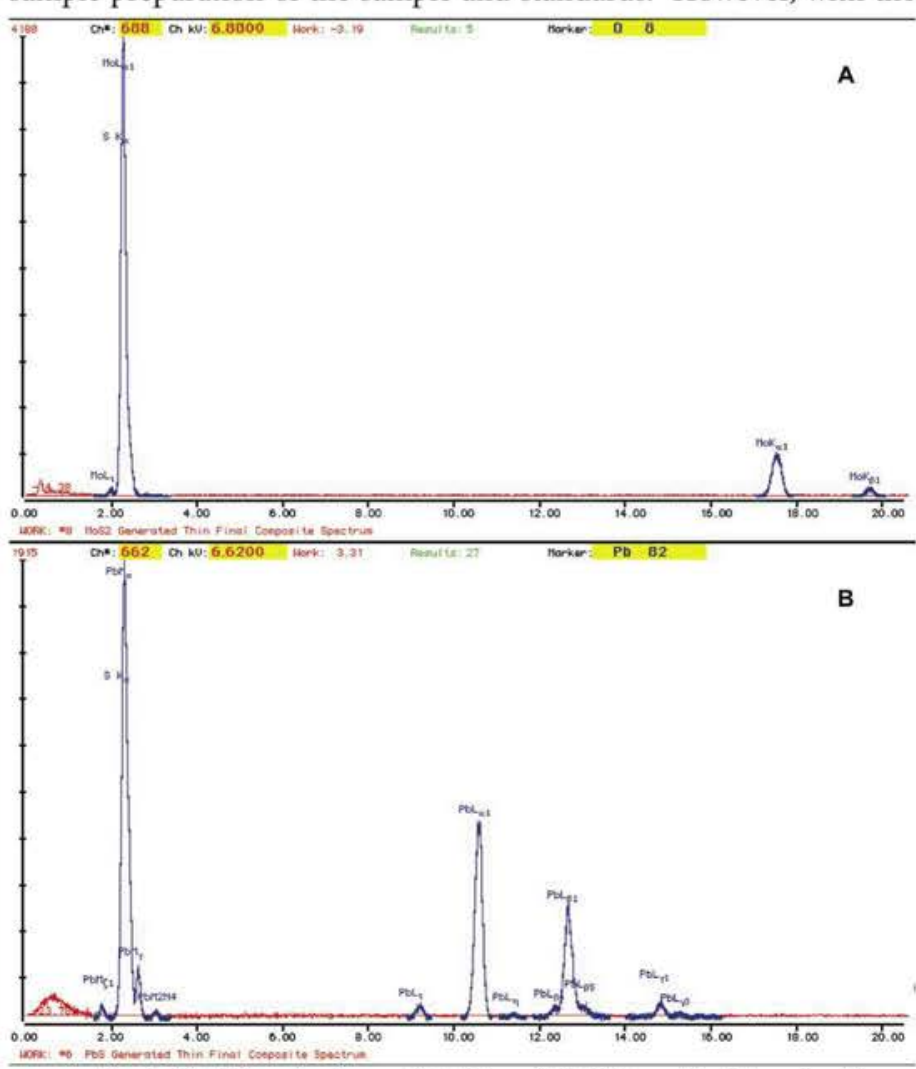

Fig. 4 XEDS spectra from A) $\mathrm{MoS}_{2}$ and B) PbS used to determine the Cliff-Lorimer k-factors.
What was reported was the ratio of the concentrations of the elements. In the paper, when there was a single phase present or the electron beam was spread out over a larger area, the values agreed fairly well with X-ray photoelectron spectroscopy (XPS) results, which report an average of the thin film composition. For this exercise, I will include the oxygen since it is only a simulation and no one will believe the results for it anyway. The AEM system modeled was a JEOL 2000FX TEM with a Kevex Quantum detector. The parameters used in the modeling were $200 \mathrm{kV}$ for accelerating voltage, $0.150 \mathrm{nA}$ for the beam current, and $300 \mathrm{~s}$ live time. All of the samples were considered to be uniform, parallel-sided, $50 \mathrm{~nm}$-thick thin films.

The first step in the process that is required is to determine the ratio of the higher energy line to the lower energy line. For lack of a better name, I will call this the elemental intensity ratio. For the same reasons that it is better to determine Cliff-Lorimer $k$-factors experimentally because of variations in TEM/XEDS systems, the elemental intensity ratios should be experimentally determined instead of accepting published values. For this system, it was easier to grind the $\mathrm{Pb}$ and Mo oxide and sulfide powders because I had them on hand. Only areas of the samples that showed kinematical convergent beam electron disks in the $200 \mathrm{keV}$ instruments were used and strongly diffracting conditions were avoided. Fig. 3 shows the modeled X-ray spectra from $\mathrm{MoO}_{3}$ and $\mathrm{PbO} 50 \mathrm{~nm}$ thick standards from DTSA. The integrated peak intensities and elemental intensity ratio values are given in Table 1. In the table, the ratio is found to be the Intensity of the lower energy line to the higher energy line, e.g. $\mathrm{R}_{\mathrm{Mo}-L / K}=\mathrm{I}_{\mathrm{Mo}-L \alpha} / \mathrm{I}_{\mathrm{Mo}-\mathrm{K \alpha}}$.

The next step is to determine the $k$-factors for the Cliff-Lorimer analysis. For this step, $\mathrm{PbS}$ and $\mathrm{MoS}_{2}$ were used as the standards. Fig. 4 shows the modeled X-ray spectra from these materials. The overlapped $\mathrm{S}$ intensities were found after subtracting the $\mathrm{Pb}-\mathrm{Ma}$ and the Mo- $L a$ lines from their convoluted peaks, respectively. To do this, the intensity of the Mo- $L \alpha$ and the $\mathrm{Pb}-M \alpha$ lines are found by using the ratio value found in the first step and then subtracting the calculated intensity from the convoluted overlapped peak. For example, the intensity of the S-K line from $\mathrm{MoS}_{2}$ is given as $\mathrm{I}_{\mathrm{S}-K}=\mathrm{I}_{(\mathrm{Mo}-L+\mathrm{S}-K)}-\mathrm{R}_{\mathrm{Mo}-L / \mathrm{K}}{ }^{*} \mathrm{I}_{\mathrm{Mo}-K \alpha}$. After this is done, the $k$-factors were determined. These results are also given in Table 1. 


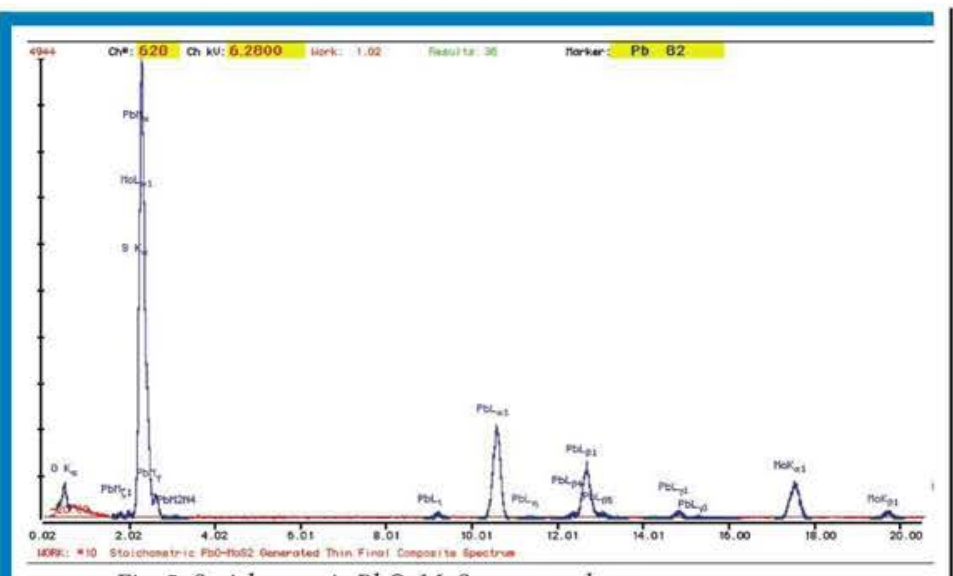

Fig. 5 Stoichometric PbO-MoS 2 as an unknown.

Now that the $k$-factors and elemental intensity ratios have been determined, the unknown can be acquired and analyzed. Fig. 5 shows the modeled spectrum from a stoichiometric $\mathrm{PbO}-\mathrm{MoS}_{2}$ film. For the case of the $\mathrm{S}-K$ line, the calculated $\mathrm{Pb}-M \alpha$ and the $\mathrm{Mo}-L \alpha$ were subtracted from the convoluted peak. The intensities and concentration ratios for the generated sample are given in Table 1. In the original study, the coatings were deposited by pulsed laser deposition. Because of this, the samples were oxygen deficient. They were also sulfur deficient, but this varied with the substrate temperature during deposition. We were primarily interested in the ratio of the elements from the deposition process. As mentioned above, we did not have a light element detector. As the results in Table 1 suggest, even at this artificial thickness of 50 $\mathrm{nm}$, there would have been tremendous absorption of the $\mathrm{O}-\mathrm{K}$ lines with this material system and with the standards.

One thing that should jump out from performing these steps is the proliferation of the accumulation of experimental errors, just on the basis of counting statistics alone. The $k$-factors and the elemental intensity ratios are formed from the ratio of two intensities and then the calculated intensity uses the product of that value with another intensity which is then subtracted from yet another intensity; then the concentration is calculated by applying the $k$-factors to two more intensities. (That sentence was as dizzying to write as it is to read.) Realistically, it means that pain must be taken to acquire many spectra from the first two steps in the process in order to minimize the contribution of experimental errors from the intensity ratio values and the $k$-factors, since those are formed from ratio of intensities whose errors are determined by counting statistics. Further, the error associated with each ratio would be found by the quadrature summation of the individual errors. I would recommend that the reader review the sections on experimental errors in any of the X-ray analysis sections of texts on electron microscopy. As riddled as this process is with the accumulation of errors in order to get the integrated peak intensity of the $\mathrm{S}-K$ line, it did provide a result that I was comfortable in reporting because it was the only method to obtain the partitioning of the elements in the samples with multiple phases and a fine microstructure. The models that were used here are ideal cases for the standards and the unknown. Overcoming the triple overlap of $\mathrm{Pb}, \mathrm{Mo}$, and $\mathrm{S}$ was an extreme example of this process. However, using it to overcome a double overlap, such as was done for the $\mathrm{MoS}_{2}$ and $\mathrm{PbS}$ samples should be relatively simple in comparison.

\section{References}

1. Characterization of Pulsed Laser Deposited $\mathrm{PbO} / \mathrm{MoS}_{2}$ by Transmission Electron Microscopy, S. D. Walck, M. S. Donley, J. S. Zabinski, and V. J. Dyhouse, I. Mat. Res., 9 (1), pp. 236-45, 1994.

2 D. B. Williams, Practical Analytical Electron Microscopy in Materials Science, Philips Electronic Instruments, Inc., Mahwah, NJ, 1984.

3 Beginner's Guide to X-Ray Analysis in an Analytical Electron Microscope: Part 2 Quantification Using Absorption and Fluorescence Corrections, N. J. Zaluzec, EMSA Bulletin, 14, (2), pp. 61-72, 1984.

4 See the website, http://www.cstl.nist.gov/div837/Division/outputs/DTSA/DTSA.htm, for a complete description of DTSA.

\section{Register now to attend ...}

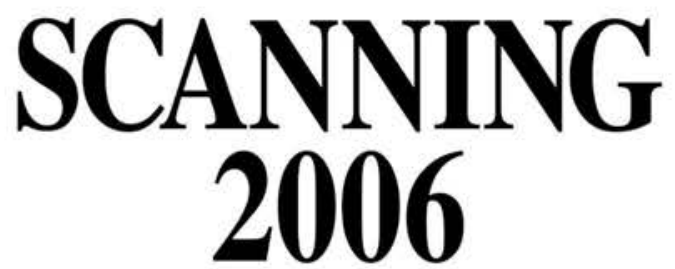

The Seventeenth Annual International

Scientific Meeting sponsored by the Foundation for Advances in

Medicine and Science, Inc.

\section{at the historic Hotel Washington and adjacent Willard Hotel in Washington, D.C.}

\author{
Tuesday, April 25 \\ Wednesday, April 26 \\ and Thursday, April 27
}

- Opening Reception at the prestigious National Press Club

- Post-meeting tour of the National Institute of Standards and Technology (NIST), Friday, April 28 (9:30 a.m. to 2:00 p.m.)

- Complimentary meeting registration to qualified area students

- Sessions and short courses on the applications of the scanning microscopies in forensics, industry, medicine, biology, nanotechnology, and other disciplines.

- Art exhibit and contest for registrants

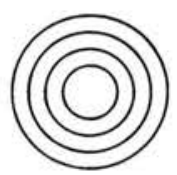

Visit www.scanning.org

for the complete program, or to register on-line.
Inquire by phone 800-443-0263 or 201-818-1010 or fax 201-818-0086 email: scanning@fams.org 